\title{
NÍVEIS SÉRICOS DE TRIIODOTIRONINA (T3) E TIROXINA (T4) EM OVELHAS COM TOXEMIA DA PRENHEZ
}

\section{(SERUM LEVELS OF TRIIODOTHYRONINE (T3) AND THYROXINE (T4) IN EWES DIAGNOSED WITH PREGNANCY TOXEMIA)}

\author{
Alexandre Tadeu Mota* Macedo, Rodolfo Cavalcanti Souto, Carla Lopes Mendonça, \\ Liliane Bezerra Lima, Pierre Castro Soares, José Augusto Bastos Afonso \\ 1 Correspondência: alexandretmota@hotmail.com:*
}

RESUMO: O presente trabalho tem por objetivo verificar os níveis séricos hormonais de triiodotironina (T3) e tiroxina (T4) em ovelhas diagnosticadas com toxemia da prenhez (TP), bem como avaliar sua participação e reflexo neste tipo de transtorno metabólico. Foram analisadas as fichas clínicas de 24 ovelhas atendidas na Clínica de Bovinos, Campus Garanhuns/UFRPE, diagnosticadas com TP, no período de 2007 a 2012. Os animais foram submetidos ao exame clínico e ultrassonográfico, seguido de coleta de sangue e urina para exames laboratoriais. As principais alterações clínicas apresentadas pelos animais foram apatia, aumento da temperatura corporal, mucosas congestas, desidratação, edema dos membros, amaurose, taquicardia, inapetência a anorexia, diminuição da motilidade ou atonia ruminal. Vieram a óbito $50 \%$ dos animais, destes $58,33 \%$ estavam hiperglicêmicos. A pesquisa de corpos cetônicos revelou um quadro de cetonúria. Quanto a glicemia, foram constatadas três condições nas ovelhas com TP. Os resultados laboratoriais revelaram valores elevados para ácidos graxos não-esterificados (AGNES) e $\beta$ hidroxibutirato (BHB). Enquanto que a média dos níveis séricos de T3 $(3,21 \mathrm{nmol} / \mathrm{L})$ e de T4 $(69,16 \mathrm{nmol} / \mathrm{L})$ nas ovelhas com TP foram consideradas como de baixas concentrações. Dessa forma, é possível concluir que a TP é caracterizada por alterações endócrinas que refletem o estado de balanço energético negativo em que as ovelhas acometidas se encontram.

Palavras-chave: corpos cetônicos; distúrbio metabólico; hormônios; ovinos

\begin{abstract}
The aims of the present study were to determine serum levels of triiodothyronine (T3) and thyroxine (T4) in ewes diagnosed with pregnancy toxemia (PT) and evaluate the participation of these hormones in this type of metabolic disorder. Analyses were performed of the clinical cases of 24 ewes diagnosed with PT and treated at the Bovine Clinic of the University Federal Rural of Pernambuco, Campus Garanhuns, from 2007 to 2012. The animals were submitted to clinical and ultrasound exams. Blood and urine were collected for the laboratory exams. The main abnormalities were apathy, elevated body temperature, congested mucosa, dehydration, edema in the limbs, amaurosis, tachycardia, inappetence or anorexia and diminished ruminal motility. The mortality rate was $50 \%$. Among the animals that died, $58.33 \%$ were hyperglycemic. The study of ketone bodies revealed ketonuria. Three conditions of glycemia were found in the ewes with PT. The laboratory findings revealed high levels of non-esterified fatty acids and $\beta$-hydroxybutyric acid. Serum levels of T3 $(3.21 \mathrm{nmol} / \mathrm{L})$ and T4 $(69.16 \mathrm{nmol} / \mathrm{L})$ were low. In conclusion, pregnancy toxemia in ewes is characterized by endocrine alterations that reflect a negative energy balance..
\end{abstract}

Key Words: hormones; ketones; metabolic disorder; ovine 


\section{INTRODUÇÃO}

A toxemia da prenhez (TP) é um distúrbio multifatorial do metabolismo energético, proteico e hormonal (Radostits et al., 2007; Santos et al., 2011). O balanço energético negativo, os transtornos glicêmicos e o aumento do catabolismo de gordura produzem a característica clínica de cetonemia, cetonúria e sinais neurológicos como depressão, inapetência, incoordenação, deficiência visual e prostração que progride e que nos casos mais severos na maioria das vezes evolui para morte (Kimberling, 1988). Ocorre usualmente no último mês de gestação, geralmente gemelar ou, ocasionalmente, de um único feto muito desenvolvido (Bruére e West, 1993; Riet-Correa et al., 2007; Campos et al., 2010; Corrêa et al., 2010).

Os eventos metabólicos centrais são a disponibilidade de glicose e a mobilização de gordura, que aumentam em função do ganho de peso dos fetos, em torno de $80 \%$, nas últimas 6 semanas de gestação (Kimberling, 1988; Smith e Sherman, 2009). Na TP, o ácido propiônico e os precursores de glicogênio derivados da dieta e das reservas do corpo são incapazes de manter os requisitos de glicose. Assim, a hipoglicemia é a primeira anomalia detectada na enfermidade. Ocorre então, a $\beta$-oxidação dos ácidos graxos como fonte de energia alternativa e, consequentemente a produção de acetil-CoA, que pode entrar no ciclo de Krebs ou pode ser convertido em corpos cetônicos. A hipercetonemia verificada é uma característica comum da doença em cabras e ovelhas, entretanto Harmeyer e Schlumbohm (2006) relataram que esta condição não representa apenas um sinal da doença, mas atua de maneira multifuncional em promover o desenvolvimento da TP. Tal fato foi justificado pela reduzida habilidade de ovelhas multíparas em final de gestação em utilizar corpos cetônicos, o que demonstra haver uma grande importância deste componente na patogênese dessa desordem metabólica (Souto et al., 2013)

Os ajustes homeostáticos que ocorrem durante a gestação dependem de mecanismos endócrinos e neuroendócrinos, dos quais os hormônios triiodotironina (T3) e tiroxina (T4) têm um importante papel regulatório (Colodel et al., 2010). Os hormônios tireoidianos (HT) exercem um profundo efeito no metabolismo e em outras funções do organismo animal (Toniollo et al., 1998). Essencialmente o T3 e T4 modulam todos os caminhos metabólicos através das alterações no consumo de oxigênio e mudanças no metabolismo de proteínas, lipídeos, carboidratos e vitaminas (Norman e Litwack, 1997; Boelaert e Franklyn, 2005). Eles estimulam tanto a síntese quanto a degradação protéica, bem como a lipogênese e a lipólise no tecido adiposo, incrementam a absorção de carboidratos pelo intestino e a produção hepática de glicose por gliconeogênese, estimulam a glicogenólise mediante secreção de insulina e ativam substratos que fazem parte da glicólise (Achmadi e Terashima, 1995; Guyton e Hall, 2002). A concentração plasmática do $\mathrm{T} 4$ foi considerada indicadora do balanço energético, do ganho de peso e da deposição de gordura, e há uma boa relação das ações do T3 com a insulina, entretanto com o glucagon os efeitos são antagônicos (Ellenberger et al., 1989; Barash et al., 1998, Squires, 2003; Kulcsár et al., 2006).

$\mathrm{Na}$ TP as alterações endócrinas refletem a severidade do balanço energético negativo, destacando-se a redução dos efeitos da insulina, T3 e T4 que têm um papel crucial na fisiopatologia desta enfermidade (Henze et al., 1998, Kulcsár et al., 2006, Santos et al., 2011, Souto et al., 2013). São poucos os relatos abordando a 
participação dos HT e seus reflexos em ovelhas acometidas com este tipo de transtorno metabólico. Neste contexto, este trabalho teve como objetivo verificar os níveis séricos hormonais de T3 e T4 em ovelhas com TP.

\section{MATERIAL E MÉTODOS}

Coleta dos dados: As informações foram colhidas dos prontuários clínicos de ovelhas atendidas na Clínica de Bovinos, Campus Garanhuns/UFRPE, diagnosticadas com TP, durante 0 período de 2007 a 2012. Incluíram-se os dados de 24 ovelhas criadas nos estados de Pernambuco e Alagoas, sendo estas das raças Santa Inês, Dorper e mestiças, com idades entre $1 \mathrm{e}$ 5 anos, peso médio de $60 \mathrm{~kg}$, em sua maioria multíparas, predominantemente submetidas ao sistema intensivo de criação. A maioria dos animais apresentava o escore corporal 3 ou superior e eram alimentadas com ração a base de concentrados. O exame clínico foi realizado segundo Radostits et al. (2007), e foram submetidas ao exame ultrassonográfico transabdominal, utilizando probe convexa de $3,5 \mathrm{mHz}$, para determinação do número de fetos e da viabilidade fetal. Este procedimento foi realizado em 13 das 24 ovelhas.

\section{Colheita das amostras:}

Amostras de sangue foram coletadas por venopunção jugular, com agulha $25 \times 8 \mathrm{~mm}$ em tubos siliconizados vacutainer sem anticoagulante para obtenção de soro. Os tubos foram centrifugados a 3500rpm por cinco minutos, as alíquotas obtidas foram acondicionadas em tubos tipo eppendorf e armazenados em ultra freezer à temperatura de $-80^{\circ} \mathrm{C}$.

Análises laboratoriais: a determinação plasmática da glicose (Labtest Diagnóstica) foi realizada de acordo com as recomendações do fabricante. Os ácidos graxos não esterificados (AGNE) e $\beta$-hidroxibutirato (BHB) foram determinados de acordo com os reagentes comerciais (Randox Laboratories Ltd). Essas análises foram realizadas na Clínica de Bovinos, Campus Garanhuns, UFRPE. As concentrações de T3 e T4 foram determinadas pelo método de eletroquimioluminescência (ECL) empregando-se 0 kit comercial da Roche Diagnostics ${ }^{\circledR}$ através do equipamento da Roche Cobas e 411 e foram efetuadas no Hospital Militar de Área do Recife - HMAR.

Análise Estatística: $A$ análise estatística dos dados foi realizada de forma descritiva, determinando as distribuições de frequências das variáveis analisadas.

\section{RESULTADOS}

Ao exame clínico, os principais achados apresentados pelas ovelhas foram: apatia, sendo que $12,5 \%$ delas já se encontravam em estado précomatoso; aumento da temperatura corpórea; mucosas congestas, desidratação, edema dos membros, amaurose, taquicardia e vasos episclerais injetados. Houve evidência de inapetência a anorexia, distensão abdominal, diminuição da motilidade ou atonia ruminal (Apêndice 1). $O$ índice de sobrevivência materno, assim como a taxa de mortalidade foram de $50 \%$. A evolução clínica nos animais que receberam alta foi de 14 dias, enquanto os animais que vieram a óbito foram de cinco dias.

O resultado da ultrassonografia revelou que em $38,46 \%$ (5/13) dos casos foi evidenciada a presença de pelo menos um feto vivo; $30,76 \%(4 / 13)$ dos casos revelaram a presença de pelo menos dois fetos vivos; em 15,38\% (2/13) das ovelhas observou-se a presença de pelo menos um feto morto, devido à ausência dos batimentos 
cardíacos; e em 7,69\% (1/13) dos casos, se evidenciou a presença de pelo menos dois fetos mortos, assim como também pôde ser observado a presença de um feto morto e um vivo, com este mesmo percentual.

Das 24 ovelhas, dez foram submetidas a cesariana $(41,67 \%)$, seis a manobra obstétrica (25\%), uma pariu normalmente e outra abortou (4,17\%), quatro vieram a óbito $(16,67 \%)$ e duas receberam alta clínica (8,33\%) antes do parto. Dos 17 partos ocorridos na Clínica de Bovinos, 14 foram múltiplos $(82,35 \%)$, sendo que em $78,57 \%$ dos casos (11/14) havia dois fetos e em $21,43 \%$ (3/14) três fetos; e três partos foram simples $(17,64 \%)$. Foram obtidos 34 fetos, através de cesariana, manobra obstétrica e parto normal, destes $58,82 \%$ (20/34) eram machos e $41,18 \%$ $(14 / 34)$ fêmeas, $55,88 \%$ dos fetos $(19 / 34)$ estavam vivos, enquanto que $44,12 \%$ deles (15/34) já se encontravam mortos. A pesquisa de corpos cetônicos na urina caracterizou um quadro de cetonúria.

Em relação à glicemia, houve três estados glicêmicos, onde $54,17 \%$ das ovelhas com TP estavam hiperglicêmicas, $\quad 25 \% \quad$ estavam normoglicêmicas e a minoria 20,83\% estava hipoglicêmica. Vieram a óbito $50 \%$ dos animais (12/24), destes 63,63 $\% \quad(7 / 12)$ estavam hiperglicêmicos (Quadro 2).

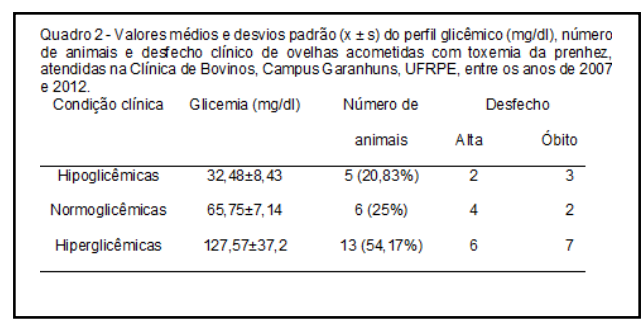

Os valores para ácidos graxos não-esterificados (AGNES) e $\beta$ hidroxibutirato (BHB) estavam elevados. Enquanto para os hormônios tireoidianos as médias das concentrações séricas de T3 $(3,21$ $\mathrm{nmol} / \mathrm{L})$ e de T4 $(69,16 \mathrm{nmol} / \mathrm{L})$ nas ovelhas com TP foram consideradas estarem baixas (Quadro 3).

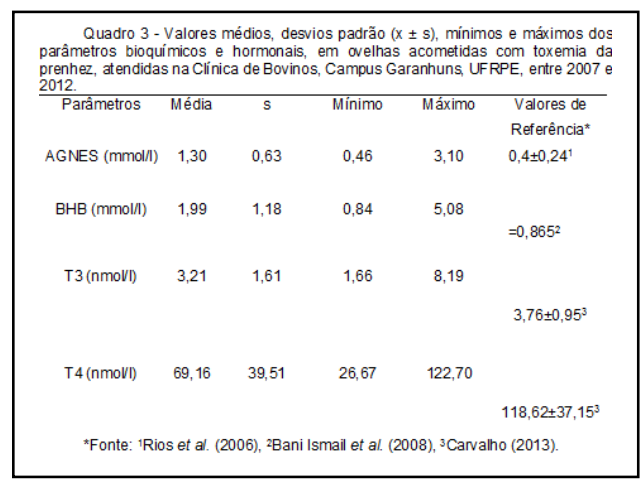

A sintomatologia clínica apresentada pelas ovelhas com TP, foi semelhante aos achados de Kimberling (1988) e Henze et al. (1998), assim como também aos de Souto et al. (2013) em seu trabalho com cabras. Outros autores como Bruére e West (1993), Andrews (1997) e Mobini et al. (2005), também relataram as manifestações nervosas como frequentes na evolução da doença, retratando-as com a gravidade dos casos. Em relação ao índice de sobrevivência materno, este foi maior quando comparado a citação de Rook (2000), que relata valores próximos a $80 \%$ na taxa de mortalidade em rebanhos acometidos, principalmente quando 0 início do tratamento é retardado e os animais se encontram numa condição pré-comatosa. A evolução clínica foi semelhante a encontrada por Henze et al. (1998) e Souto et al. (2013), em ovelhas e cabras acometidas por TP, e justifica a evolução clínica mais longa para os pacientes que obtiveram alta, e que se deve provavelmente a intervenção terapêutica mais precoce diante dos sinais clínicos apresentados.

Quanto aos diferentes estados glicêmicos, em que a hiperglicemia 
ocorreu em mais de $50 \%$ dos casos, esse achado discorda do que foi relatado por Ranaweera (1980), Ford et al. (1990) e Scott et al. (1995), onde a maior ocorrência de TP foi em ovelhas hipoglicêmicas. Porém estão de acordo com Henze et al. (1998), Barakat et al. (2007), Lima et al. (2012) e Souto et al. (2013), por não encontrarem apenas uma condição de glicemia, mas três estados glicêmicos nas cabras e ovelhas acometidas com TP. A hiperglicemia ocorre pela condição de estresse das ovelhas, devido aos elevados índices de cortisol, que resultam numa gliconeogênese, e que se tornam mais acentuados quando ocorre a morte dos fetos (Ford et al., 1990). Outro fator são os baixos níveis de insulina e elevados de AGNE, este último evidente nos achados, cuja condição tem sido postulada como resultado da criação de um ambiente no qual se suporta a mínima utilização de glicose pelos tecidos periféricos e seu máximo uso pelo útero grávido, ou seja, a sensibilidade dos tecidos periféricos para insulina está reduzida e a mobilização de tecido adiposo para produzir AGNE como fonte alternativa de energia para a ovelha está elevada, esta alteração pode reforçar a hipótese de que ocorra uma insuficiência na regulação hormonal do metabolismo de lipídeos e da glicoestase como causa da TP, durante a sua evolução nas ovelhas (Lomax et al., 1979; Pethick et al., 1983; Sigurdsson, 1991; Peterson et al., 1993; Schlumbohm e Harmeyer, 2008).

O aumento nas concentrações de AGNE e BHB reflete a condição de balanço energético negativo, uma vez que são os principais indicadores da lipomobilização em ruminantes (Sargison et al., 1994; González et al., 2000). Esse achado está de acordo com Scott et al. (1995), Henze et al. (1998), Schlumbohm e Harmeyer (2004) e Souto et al. (2013), que justificam esse aumento ocorrer em virtude da lipólise que há em função da alta demanda por energia nas últimas semanas de gestação com o rápido crescimento dos fetos. Para Santos et al. (2011), o aumento sanguíneo da concentração BHB (hipercetonemia) está relacionado com a gravidade da doença.

Quanto a concentração dos hormônios T3 e T4 os resultados se assemelham aos relatados por Kulcsár et al. (2006), Gupta et al. (2008) e Carvalho (2013) que encontraram uma redução na função destes hormônios em cabras e ovelhas acometidas por TP. Esta condição pode ser explicada pelo elevado grau de inativação destes hormônios, que ocorre nos tecidos periféricos e/ou alternativamente por uma diminuição na capacidade de ativação do T4, diminuindo a transformação do T4 para T3. Estas modificações representam um importante mecanismo de adaptação a condição de balanço energético negativo (Pethes et al., 1985, Huszenicza et al., 2002, Lents et al., 2005, Huszenicza et al., 2006).

\section{CONCLUSÃO}

Diante dos achados obtidos neste trabalho podemos concluir que a toxemia da prenhez em ovelhas acarreta alterações marcantes no metabolismo dos hormônios triiodotironina (T3) e tiroxina (T4), refletindo a severa complexidade do transtorno metabólico desencadeado por ela.

\section{REFERÊNCIAS}

ACHMADI, J.; TERASHIMA, Y. The effect of propylthiouracil-induced low thyroid function on secretion response and action of insulin in sheep. Domestic. Anim. Endocrinol., Butterworth, v. 12, n. 2, p. 157-166, apr. 1995.

ANDREWS, A. Pregnancy toxaemia in the ewe. [S.I.: s.n.], 1997, 306-312 p. 
BANI ISMAIL Z.A., AL MAJALI A.M., AMIREH F. \& AL-RAWASHDEH O.F. Metabolic profiles in goat does in late pregnancy with and without subclinical pregnancy toxemia. Veterinary Clinical Pathology. Jordan: Irbid, 4(37):434437p, 2008.

BARAKAT, S.E.M.; AL-BHANASAWI, N.M.; ELAZHARI, G.E. e BAKHIET A.O. Clinical and serobiochemical studies on naturally - occurring pregnancy toxaemia in Shamia goats. J. Anim. Vet. Adv. 6(6):768-772, 2007.

BARASH, H.; AHANONI, Y.; BROSH, A.; HOLZER, Z. Effects of low energy diets followed by a compensatory diet on body weight gain and plasma hormone concentrations in bull calves. Journal of Dairy Science, v. 81, n.1, p. 50-254, 1998.

BOELAERT, K.; FRANKLYN, J.A. Thyroid hormone in health and disease. Journal of Endocrinology, v. 187, p. 1-15, 2005.

BRUÉRE A.N.; WEST D.M. The Sheep: Health, disease and production. Massey University, Palmerston North, New Zealand. 1993, 182p.

CAMPOS, A.G; AFONSO, J.A.B; SANTOS, R.A; MENDONÇA, C.L; GUIMARÃES, J.A. Estudo Clínico-Laboratorial da Toxemia da Prenhez em Ovelhas: Análise Retrospectiva. Ci. Anim. Bras., Goiânia, v. 11, n. 3, p. 623-628, jul./set. 2010.

CARVALHO, C.C.D. Indicadores Preditivos para - Diagnóstico e Controle da Toxemia da Prenhez em Ovelhas. Recife, 2013. 82-83p; 138-139p. Tese (Doutorado em Ciência Veterinária).

COLODEL, M.M.; MARTINS, E.; MARTINS, V.M.V.; MARQUES JÚNIOR, A.P. Serum concentration of thyroid hormones in crioula lanada serrana ewes in gestation and lactation. Archivos Zootecnia, v.59, n.228, p.509-517, 2010.

CORRÊA, M.N; GONZÁLEZ, F.H.D; SILVA, S.C. Transtornos Metabólicos nos Animais Domésticos. 1. ed. Pelotas: Editora e Gráfica Universitária. 2010. 148p.

ELLENBERGER, M.A.; JOHNSON, D.E.; CARSTENS, G.E.; HOSSNER, K.L.; HOLLAND, M.D.; NETT, T.M.; NOCKELS, C.F. Endocrine and metabolic changes during altered growth rates in beef cattle. Journal Animal Science, v. 67, n.6, p.1146-1454, 1989.

FORD, E.J.H.; EVANS, J.; ROBINSON, I. Cortisol in pregnancy toxaemia of sheep. British Veterinary Journal, v. 146, p. 539-542, 1990.

GONZÁLEZ, F.H.D.; BARCELLOS, J.O.; PATIÑO, H.O.; RIBEIRO, L.A. Perfil meta-bólico em ruminantes: seu uso em nutrição e doenças nutricionais. 1. ed. Porto Alegre: Grá ffica da Universidade Federal do Rio Grande do Sul. 2000. 108p.

GUPTA, V.K.; SHARMA, S.D; VIHAN, V.S.; KUMAR, A. Serum enzymes and thyroid hormones in sub-clinical ketosis in goats and sheep reared under organized farming system. Journal Animal Science, 78 (11): 1199-1201p, 2008.

GUYTON, A. C.; HALL, J. E. Tratado de Fisiologia Médica: os hormônios metabólicos da tireóide 10. ed., Rio de Janeiro: Guanabara Koogan. 2002. 802-812p.

HARMEYER, J.; SCHLUMBOHM, C. Pregnancy impairs ketone body disposal in late gestating ewes: Implications for onset of pregnancy toxaemia. Veterinary Science. 81:254-264, 2006.

HENZE, P.; BICKHARDT, K.; FUHRMANN, H.; SALLMANN, H.P. Spontaneous pregnancy toaxemia (Ketosis) in sheep and the role of insulin. J. Am. Vet. Med. Assoc. 45:225-226, 1998.

HUSZENICZA, G.; KULCSÁR, M.; RUDAS, P. Clinical endocrinology of thyroid gland function in ruminants: a review of literature. Vet. Med. Cz. 47, 191-202p, 2002.

HUSZENICZA, G.; KULCSÁR, M.; KÓRÓDI, P.; BARTYIK, J.; RUDAS, P.; RIBICZEY-SZABÓ, P.; NIKOLIÉ, J.A.; SAMANE, H.; IVANOV, I.; GVOZDIÉ, D. Adrenocortical and thyroid function, hormone and metabolite profiles and the onset of ovarian cyclicity in dairy cows suffering from various forms of ketosis. Acta Vet. Beograd 56, 25-36p, 2006.

KIMBERLING, C.V. Jensen and Swift's Diseases of Sheep. Philadelphia: Lea \& Febiger, 1988. $23 p$.

KULCSÁR, M.; DANKO, G.; DELAVAUD, C.; MIRCU, C.; NIKOLIC, A.J; GASPÁRDY, A.; CERNESCU, H.; CHILLIARD, Y.; CSEH, S.; RUDAS, P.; HUSZENICZA, G. Endocrine characteristics of late pregnant hyperketonaemic ewes and their reproductive performance following the induction of ovarian cyclicity out of the breeding season. Acta Veterinaria Hungarica 55 (2), 235-249p, 2006.

LENTS, C.A.; WETTEMAN, R.P.; WHITE, F.J.; RUBIO, I.; CICCIOLI, N.H.; SPICER, L.J.; KEISLER, D.H.; PAYTON, M.E. Influence of nutrient intake and body fat on concentrations of insulin-like growth factor-1, insulin, thyroxine and leptin in plasma of gestating beef cows. J. Anim. Sci. 83, 586-596p, 2005.

LIMA, M.S.; PASCOAL, R.A.; STILWELL, G.T. Glycaemia as a sign of the viability of the 
foetuses in the last days of gestation in dairy goats with pregnancy toxaemia. Irish Veterinary Journal. 65p, 2012.

LOMAX, M.A.; BAIRD, G.D.; MALLINSON, C.B.; SYMONDS, H.W. Differences between lactating and non-lactating dairy cows in concentration and secretion rate of insulin. Biochem. J. 180:281-289, 1979.

MOBINI, S.; HEATH, A.M.; PUGH, D.G. Teriogenologia de Ovinos e Caprinos. In: Pugh, D.G. Clínica de ovinos e caprinos. $1^{a}$ ed. São Paulo: Roca, 2005. P.145-208.

NORMAN, A.W.; LITWACK, G. Thyroid Hormones. 2. ed. San Diego: Academic Press, 1997. 169-191p.

PETHES, G.; BOKORI, J.; RUDAS, P.; FRENYP, V.L.; FEKETE, S. Thyroxin, triiodothyronine, reverse-triiodothyronine, and other physiological characteristics of parturient cows fed restricted energy. J. Dairy Sci. 68, 1148-1154p, 1985.

PETTERSON, J.A.; DUNSHEA, F.R.; EHRHARDT, R.A.; BELL, A.W. Pregnancy and undernutrition alter glucose metabolic responses to insulin in sheep. J. Nutr. 123:1286-1295p, 1993.

PETHICK, D.W.; LINDSAY, D.B.; BARKER, P. J.; NORTHROP, A. J. The metabolism of circulating non-esterified fatty acids by the whole animal, hind-limb muscle and uterus of pregnant ewes. Br. J . Nutr. 49:129-143p, 1983.

RADOSTITS, O.M.; GAY, C.C.; HINCHCLIFF, K.W.; CONSTABLE, P.D. Veterinary Medicine: A textbook of the diseases of cattle, horses, sheep, pigs and goats. 10th ed. W.B. Saunders Edinburgh, 2007. 1668p.

RANAWEERA, A. Biochemical aspects of pregnancy in ewes: a review. Ceylon Veterinary Journal, v. 28, p. 1-4, 1980.

RIET-CORREA, F.; SCHILD, A.L.; LEMOS, R.A.A.; BORGES, J.R.J. Doenças de ruminantes e equídeos. 3ed. Santa Maria: Paloti, 2007. 281286p.

RIOS, C.; MARÍN, M.P.; CATAFAU, M.; WITTWER F. Concentraciones sanguíneas de $\beta$ - hidroxibutirato, NEFA, colesterol y urea en cabras lecheras de três rebaños com sistemas intensivos de producción y surelaciónconel balance nutricional. Arch. Med. Vet. 38(1): 1923p, 2006.

ROOK, J. S. Pregnancy toxemia of ewes, does, and beef cows. Veterinary Clinics of North America: Food Animal Practice, v.16, n. 2, 293317p, 2000.
SANTOS, F.C.O.; MENDONÇA, C.L.; FILHO, A.P.S.; CARVALHO, C.C.D.; SOARES, P.C.; AFONSO, J.A.B. Indicadores bioquímicos e hormonais de casos naturais de toxemia da prenhez em ovelhas. Pesq. Vet. Bras. 31(11):974-980, 2011.

SARGISON, N.D.; SCOTT, P.R.; PENNY, C.D.; PIRIE R.S.; KELLY, J.M. Plasma enzymes and metabolites as potential prognostic indices of ovine pregnancy toxaemia: A preliminary study. Brit. Vet. J. 150(3):271-277p, 1994.

SCHLUMBOHM, C.; HARMEYER, J. Hyperketonemia impairs glucose metabolism in pregnant and nonpregnant ewes. J. Dairy Sci. 87:350-358p, 2004.

SCHLUMBOHM, C.; HARMEYER, J. Twinpregnancy increases susceptibility of ewes to hypoglycaemic stress and pregnancy toxaemia. Res. Vet. Sci. 84:286-299p, 2008.

SCOTT, P.R.; SARGISON, N.D.; PENNY, C.D.;, PIRIE R.S.; KELLY J.M. Cerebrospinal fluid and plasma glucose concentrations of ovine pregnancy toxaemia cases, inappetant ewes and normal ewes during late gestation. Brit. Vet. J. 151:39-44p, 1995.

SIGURDSSON H. Metabolic disorders in ewes during late pregnancy. Iceland Agric. Sci. 5:2531p, 1991.

SMITH, M.C.; SHERMAN, D. Goat Medicine. 2nd ed. Lea and Febiger, Philadelphia: 2009. $871 \mathrm{p}$.

SOUTO, R.J.C.; AFONSO, J.A.B.; MENDONÇA, C.L.; CARVALHO, C.C.D.; FILHO A.P.S.; CAJUEIRO, J.F.P.; LIMA, E.H.F.; SOARES, P.C. Achados Bioquímicos, Eletrolíticos e Hormonais de Cabras Acometidas com Toxemia da Prenhez. Pesq. Vet. Bras. 33(10):1174-1182, 2013.

SQUIRES, E.J. Applied Animal Endocrinology. Massachusetts: CABI publishing USA, 2003, 234p.

TONIOLLO, G.H.; VICENTE, W.R.R.; OLIVEIRA, C.A.; MALHEIROS, E.B.; CARVALHO, M.B. Avaliação dos níveis séricos de triiodotironina (T3) e tiroxina (T4) durante 0 ciclo estral em marrãs (Sus scrofa domestica Linnaeus, 1758). Braz. J. vet. Res. anim. Sci., São Paulo, v. 35, n. 5, p. 210-214, 1998. 
Apêncdice 1 - Principais sinais clínicos observados em ovelhas acometidas com toxemia da prenhez, atendidas na Clínica de Bovinos, Campus Garanhuns, UFRPE, entre 2007 e 2012.

\begin{tabular}{llcc}
\hline Características & Achados clínicos & \multicolumn{2}{c}{ Número de animais } \\
\cline { 2 - 4 } & & $\mathrm{FA}^{*}$ & $\mathrm{FR}^{\star \star}(\%)$ \\
\hline Atitude & Estação & 14 & 58,33 \\
& Decúbito & 10 & 41,67 \\
Comportamento & Calmo & 10 & 41,67 \\
& Apático & 10 & 41,67 \\
& Pré-comatoso & 3 & 12,5 \\
& Ativa & 1 & 4,17 \\
Escore corporal & 1 & 1 & 4,17 \\
& 2 & 4 & 16,67 \\
& 3 & 6 & 25 \\
& 4 & 7 & 29,17 \\
& 5 & 6 & 25
\end{tabular}

$\begin{array}{llll}\text { Temperatura corpórea } & \leq 39,5^{\circ} \mathrm{C} & 8 & 33,33 \\ & \geq 39,5^{\circ} \mathrm{C} & 16 & 66,67 \\ \text { Mucosas } & \text { Congestas } & 19 & 79,17 \\ & \begin{array}{l}\text { Pálidas } \\ \text { Rosa-pálidas }\end{array} & 2 & 8,33 \\ & & 3 & 12,5 \\ \text { Grau de desidratação } & \text { Ausente } & 3 & 12,5 \\ & \text { Leve } & 2 & 8,33 \\ & \text { Moderado } & 15 & 62,5 \\ & \text { Grave } & 4 & 16,67 \\ \text { Linfonodos externos } & \text { Sem alterações } & 14 & 58,33 \\ & \text { Hipertrofiados } & 10 & 41,67 \\ \text { Edema nos membros } & \text { Ausente } & 18 & 75 \\ & \text { Membros pélvicos } & 5 & 20,83 \\ & \text { Quatro membros } & 1 & 4,17 \\ \text { Alterações visuais } & \text { Amaurose } & 3 & 12,5 \\ & \text { Opacidade(córnea) } & 2 & 8,33 \\ \text { Frequência } & & & \\ \text { respiratória } & 24-36 & 15 & 62,5 \\ & >36 & 6 & 25 \\ \text { Intensidade } & <24 & 3 & 12,5 \\ \text { respiratória } & \text { Eupnéica } & 8 & 33,33 \\ & \text { Polipnéica } & 12 & 50\end{array}$




\begin{tabular}{|c|c|c|c|}
\hline & Oligopnéica & 4 & 16,67 \\
\hline Frequência cardíaca & $\begin{array}{l}60-90 \\
>90 \\
<60\end{array}$ & $\begin{array}{l}1 \\
20 \\
3\end{array}$ & $\begin{array}{l}4,17 \\
83,33 \\
12,5\end{array}$ \\
\hline Claudicação & $\begin{array}{l}\text { Ausente } \\
\text { Presente }\end{array}$ & $\begin{array}{l}19 \\
5\end{array}$ & $\begin{array}{l}79,17 \\
20,83\end{array}$ \\
\hline $\begin{array}{l}\text { Preenchimento dos } \\
\text { vasos episclerais }\end{array}$ & $\begin{array}{l}\text { Sem alterações } \\
\text { Injetados } \\
\text { Vazios }\end{array}$ & $\begin{array}{l}1 \\
22 \\
1\end{array}$ & $\begin{array}{l}4,17 \\
91,67 \\
4,16\end{array}$ \\
\hline Dispnéia & $\begin{array}{l}\text { Ausente } \\
\text { Presente }\end{array}$ & $\begin{array}{l}17 \\
7\end{array}$ & $\begin{array}{l}70,83 \\
29,17\end{array}$ \\
\hline Motilidade ruminal & $\begin{array}{l}\text { Sem alteração } \\
\text { Diminuído } \\
\text { Ausente }\end{array}$ & $\begin{array}{l}6 \\
13 \\
5\end{array}$ & $\begin{array}{l}25 \\
54,17 \\
20,83\end{array}$ \\
\hline Abomaso & $\begin{array}{l}\text { Fisiológico } \\
\text { Hipomotílico }\end{array}$ & $\begin{array}{l}12 \\
12\end{array}$ & $\begin{array}{l}50 \\
50\end{array}$ \\
\hline Intestinos & $\begin{array}{l}\text { Fisiológico } \\
\text { Hipomotílico }\end{array}$ & $\begin{array}{l}12 \\
12\end{array}$ & $\begin{array}{l}50 \\
50\end{array}$ \\
\hline Apetite & $\begin{array}{l}\text { Presente } \\
\text { Caprichoso } \\
\text { Ausente }\end{array}$ & $\begin{array}{l}11 \\
3 \\
10\end{array}$ & $\begin{array}{l}45,83 \\
12,5 \\
41,67\end{array}$ \\
\hline $\begin{array}{l}\text { Abdômen } \\
\text { Tensão Abdominal }\end{array}$ & $\begin{array}{l}\text { Sem alterações } \\
\text { Distendido } \\
\text { Fisiológica } \\
\text { Aumentada }\end{array}$ & $\begin{array}{l}7 \\
17 \\
6 \\
18\end{array}$ & $\begin{array}{l}29,17 \\
70,83 \\
25 \\
75\end{array}$ \\
\hline
\end{tabular}

${ }^{*}$ FA - Frequência Absoluta; **FR - Frequência Relativa 